\title{
Phase II study of sequential chemoradiotherapy with L-asparaginase, dexamethasone, ifosfamide, cisplatin, and etoposide (DICE-L) in the early stage of extranodal natural killer (NK)/T-cell lymphoma
}

\author{
Yao Zhang ${ }^{1,2}$, Yizhen Liu ${ }^{1,2}$, Zuguang Xia ${ }^{1,2}$, Jia Jin ${ }^{1,2}$, Kai Xue ${ }^{3}$, Jiachen Wang ${ }^{1,2}$, Hui Sun ${ }^{1,2}$, Fangfang Lv $^{1,2}$, \\ Xiaojian Liu ${ }^{1,2}$, Junning Cao ${ }^{1,2}$, Xiaonan Hong ${ }^{1,2}$, Ye Guo ${ }^{4}$, Xuejun $\mathrm{Ma}^{5}$, Qunling Zhang ${ }^{1,2}$ \\ ${ }^{1}$ Department of Medical Oncology, Fudan University Shanghai Cancer Center, Shanghai, China; ${ }^{2}$ Department of Oncology, Shanghai Medical \\ College, Fudan University, Shanghai, China; ${ }^{3}$ Department of Hematology, Ruijin Hospital Affiliated to Shanghai Jiao Tong University School of \\ Medicine, Shanghai, China; ${ }^{4}$ Department of Medical Oncology, Shanghai East Hospital, Tongji University School of Medicine, Shanghai, China; \\ ${ }^{5}$ Department of Radiation Oncology, Fudan University Shanghai Cancer Center, Shanghai, China \\ Contributions: (I) Conception and design: Q Zhang, X Ma, Y Guo; (II) Administrative support: Q Zhang; (III) Provision of study materials or patients: \\ All authors; (IV) Collection and assembly of data: All authors; (V) Data analysis and interpretation: Y Zhang, Y Liu; (VI) Manuscript writing: All \\ authors; (VII) Final approval of manuscript: All authors. \\ Correspondence to: Qunling Zhang. Department of Medical Oncology, Fudan University Shanghai Cancer Center, 270 Dongan Road, Xuhui District, \\ Shanghai 200032, China. Email: zhangqunling@fudan.edu.cn; And Xuejun Ma. Department of Radiation Oncology, Fudan University Shanghai \\ Cancer Center, Shanghai 200032, China. Email: chateauma@hotmail.com.
}

Background: To explore a more effective treatment strategy for newly diagnosed stage I and II extranodal natural killer/T-cell lymphoma (ENKTL), nasal type, we conducted a prospective phase II study of sequential chemoradiotherapy with the L-asparaginase, dexamethasone, ifosfamide, cisplatin, and etoposide (DICE-L) regimen.

Methods: Patients with newly diagnosed stage I and II ENKTL in the upper-aerodigestive tract were enrolled. Treatment was comprised of up to 4 cycles of DICE-L followed by 50 Gy of intensity modulated radiation therapy (IMRT) to the involved field. The primary endpoint was the complete response (CR) rate. The secondary endpoints were the objective response rate (ORR), the 5-year overall survival (OS) rate, the 5-year progression-free survival (PFS) rate, and safety.

Results: A total of 81 patients were enrolled from June 2009 to May 2012 in Shanghai Cancer Hospital. Among these patients, 68 patients achieved CR and 1 patient achieved partial response (PR). The CR rate was $84 \%$, and the ORR was $85.2 \%$. With a median follow up of 88.1 months, the 5 -year OS and 5 -year PFS rates were $82.4 \%$ and $63.4 \%$, respectively. The most common adverse events were grade 3 to 4 neutropenia $(73.5 \%)$ and febrile neutropenia $(21 \%)$.

Conclusions: Sequential chemoradiotherapy using DICE-L followed by radiotherapy is an effective treatment modality for stage I to IIE ENKTL and is safe with acceptable toxicity.

Keywords: Extranodal natural killer/T-cell lymphoma (ENKTL); sequential chemoradiotherapy; dexamethasone, ifosfamide, cisplatin, and etoposide (DICE); L-asparaginase

Submitted May 25, 2021. Accepted for publication Jul 22, 2021.

doi: 10.21037/atm-21-3525

View this article at: https://dx.doi.org/10.21037/atm-21-3525 


\section{Introduction}

Extranodal natural killer (NK)/T-cell lymphoma (ENKTL), nasal type is a distinct entity of aggressive non-Hodgkin's lymphoma, the prevalence of which is high in East Asia and Latin America, especially in China, Japan, and Korea $(1,2)$. ENKTL often involves extranodal sites, such as the nasal/ paranasal area, skin, gastrointestinal tract, eye, lung, or soft tissue, but most ENKTL occurs in the nasal/paranasal area. The association between ENKTL and Epstein-Barr Virus (EBV) infection has been confirmed in early studies (3). Most patients present with localized disease (stage IE, IIE), resulting in symptoms of nasal obstruction, epistaxis, and/ or a destructive mass (4-7). Therefore, local and systemic treatment are equally important in ENKTL therapy.

The standard treatment modality for newly diagnosed early stage ENKTL remains to be established. Radiotherapy has an important role in the management of early stage ENKTL. A prospective study showed that radiotherapy combined with chemotherapy improved complete response (CR), 5-year progression-free survival (PFS), and overall survival (OS). The 5 -year OS rates were $86 \%, 45 \%$, and $64 \%$ in the chemoradiotherapy, chemotherapy alone, and radiotherapy alone groups, respectively (8).

However, which regimens have the higher response rates remains unclear. Anthracycline-based regimens were the mainstay of treatment in the past, with a 5 -year OS of about $49.5 \%$, due to the high expression of P-glycoprotein in tumor cells (9). The clinical outcome of ENKTL has improved substantially as a result of the use of nonanthracycline-based chemotherapies which contain drugs that are not a substrate for P-glycoprotein (10). In 2 prospective studies, concurrent radiotherapy or adjuvant chemotherapy with a regimen comprising of dexamethasone, etoposide, ifosfamide, and carboplatin or cisplatin (DICE) had a promising 5 -year OS of $73 \%$ (11). However, the hematological and non-hematological toxicity increased significantly with radiotherapy and chemotherapy treatment at the same time. Sequential chemoradiotherapy including 6 cycles of chemotherapy may be a good choice to reduce toxicity without losing efficacy.

L-asparaginase is an enzyme that inhibits serum L-asparagine and shows anti-tumor activity in T-cell lymphoma including ENKTL. In patients with refractory or relapsed ENKTL, L-asparaginase-based regimens have more than $78 \%$ outstanding response rates (12). L-asparaginase has also been combined with different regimens in sequential chemoradiotherapy. In a prospective study with reduced chemotherapy cycles, it showed exciting results, with a CR rate of $87 \%$ and a 5 -year PFS rate of $73 \%$ (11). L-asparaginase combined regimens may decrease toxicity by reducing the chemotherapy cycles.

To further improve the efficacy and reduce the toxicity in stage I/II ENKTL patients, we designed this prospective phase II study, which consisted of only 4 cycles of L-asparaginase, dexamethasone, ifosfamide, cisplatin, and etoposide (DICE-L) followed by intensity modulated radiation therapy (IMRT) for stage IE or IIE ENKTL patients. We present the following article in accordance with the TREND reporting checklist (available at https:// dx.doi.org/10.21037/atm-21-3525).

\section{Methods}

\section{Participants and eligibility criteria}

A total of 81 patients were enrolled from June 2009 to May 2012 in Shanghai Center Hospital. The eligibility criteria included patients who had a biopsy-proven diagnosis of ENKTL, were 14-75 years old, had previously untreated stage I/II NK/T-cell lymphoma in the upper-aerodigestive tract, had a measurable disease, and had an Eastern Cooperative Oncology Group (ECOG) performance status of $0-2$. Patients also had adequate hematological function, renal function, and hepatic function, and a life expectancy of more than 3 months. At least 1 measurable lesion as evidenced by CT scan or MRI was required. All patients provided written informed consent. The exclusion criteria were patients who had a history of other malignancies except cured basal cell carcinoma of the skin and carcinoma in-situ of the uterine cervix, serious uncontrolled disease, incurrent infection, and evidence of CNS metastasis. Patients who were pregnant or lactating were also excluded. The study was conducted in accordance with the principles of the Declaration of Helsinki (as revised in 2013). This study was approved by the institute review board of Shanghai Cancer Center of Fudan University (No. 090674-1) and was first registered on July $7^{\text {th }}, 2009$. The NCT number is NCT00933673 (https://clinicaltrials.gov/ct2/show/ NCT00933673, first posted: July $7^{\text {th }}, 2009$ ).

\section{Interventions}

The patients received up to 4 cycles of DICE-L chemotherapy every 3 weeks in hospital. The intervention was delivered in the inpatient ward. The chemotherapy 
regimen consisted of L-asparaginase $6,000 \mathrm{IU} / \mathrm{m}^{2}$ from day 1 to 4 , dexamethasone $40 \mathrm{mg}$ from day 1 to 4 , ifosfomide $1,200 \mathrm{mg} / \mathrm{m}^{2}$ from day 1 to 4 (400 $\mathrm{mg}$ of mesna was also given at $0,4,8$ hours after each ifosfomide infusion), etoposide $60 \mathrm{mg} / \mathrm{m}^{2}$ from day 1 to 4 , and cisplatin $20 \mathrm{mg} / \mathrm{m}^{2}$ from day 1 to 4 . If diseases did not progress, the patients were treated with IMRT. Primary IMRT was delivered with a 6-MeV linear accelerator using 3D conformal or intensity modulated treatment planning. The IMRT dose was 50 gray in 25 fractions, with 2 gray a day and 5 fractions per week.

\section{Evaluation}

Baseline evaluation was performed 14 days or less before the treatment. A physical examination, complete blood count, serum biochemistry with lactate dehydrogenase (LDH) and amylase, electrocardiogram, computed tomography (CT) scan or magnetic resonance imaging (MRI) of the involved lesions, bone marrow aspiration, and positron emission tomography (PET) were performed before treatment and at the end of radiotherapy. Complete blood count, serum biochemistry with LDH and amylase, and electrocardiogram was performed before every cycle of chemotherapy. All these examinations were repeated every 3 to 6 months to monitor disease progression.

\section{Assessment of efficacy}

The primary end point was the complete remission (CR) rate. The secondary end points were objective response rate (ORR), PFS, and OS. Efficacy was assessed every 2 cycles of chemotherapy and at the end of radiotherapy by nasal cavity MRI and CT scanning of the involved disease. A PET-CT scan was performed before chemotherapy and 3 months after radiotherapy. Otolaryngologic examination was required to confirm CR. Response was assessed according to the Revised Response Criteria (13) for Lymphoma, and complete remission (CR) was defined as no evidence of residual disease, partial response (PR) was defined as at least a $50 \%$ reduction in the sum of the product of the greatest diameters (SPD), progressive disease (PD) was the presence of any new lesion or an increase by more than $50 \%$ of previously involved sites, and stable disease (SD) was defined as failure to attain CR/PR or PD.

\section{Safety}

Adverse events were assessed at each cycle and graded according to the National Cancer Institute Common Toxicity Criteria, version 4.0 from the beginning of chemotherapy to 1 month after the last study treatment.

\section{Statistical analysis}

Data were analyzed by IBM SPSS Statistics 23.0 software. The baseline characteristics and the impact of different factors on response were evaluated by the chi-square test. PFS and OS were calculated by the Kaplan-Meier and life table methods. The differences were tested by the log-rank test. A two-sided $\mathrm{P}$ value of less than 0.05 was considered significant.

According to previous studies, the CR rate for untreated early stage ENKTL with DICE was approximately $30 \%$ (14). We expected that the CR rate would be increased by $15 \%$ with DICE-L. The trial was designed with a statistical power of $80 \%$, with a one-sided, type I error of $5 \%$. The number of eligible patients required for this study was calculated to be 76 .

\section{Results}

\section{Patient characteristics}

A total of 81 patients were enrolled in this prospective study between June 2009 and May 2012 in Shanghai Cancer Hospital. The characteristics of the patients are listed in Table 1. The median age was 47 years (range, $16-73$ years), and 71 patients (87.6\%) were younger than 60 years old. There was a male predominance, with a male to female ratio of $3: 1$. All patients were stage IE or IIE, with 20 stage IE patients and 30 stage IIE patients. Ten patients (12.4\%) had elevated LDH, and 39 patients (48.1\%) presented with B symptoms at diagnosis. Most patients $(93.8 \%)$ were in the low-risk categories of the International Prognostic Index (IPI). However, 13 patients (16.05\%) belonged to group III or IV of the NK/T-cell lymphoma prognostic index (NKPI).

\section{Treatment}

Most patients $(57 / 81,70.4 \%)$ completed 4 courses of chemotherapy, while 10 patients $(12.4 \%)$ discontinued due to adverse events after less than 2 cycles of chemotherapy. A total of 56 patients (69\%) experienced dose reduction due to adverse events, especially hematological toxicities, and 76 patients $(92.7 \%)$ completed the planed radiotherapy. 


\section{Page 4 of 9}

Table 1 Baseline patient characteristics $(\mathrm{N}=81)$

\begin{tabular}{|c|c|}
\hline Characteristic & No. of patients (\%) \\
\hline Age, years (median 47), range & $16-73$ \\
\hline Age $>60$ years & $10(12.40)$ \\
\hline \multicolumn{2}{|l|}{ Sex } \\
\hline Male & $61(75.30)$ \\
\hline Female & $20(24.70)$ \\
\hline Elevated LDH & $10(12.40)$ \\
\hline B symptoms & $39(53.10)$ \\
\hline \multicolumn{2}{|l|}{ IPI } \\
\hline 0 & $57(70.40)$ \\
\hline 1 & $19(23.50)$ \\
\hline$\geq 2$ & $5(6.20)$ \\
\hline \multicolumn{2}{|l|}{ NKPI } \\
\hline 0 & $32(39.51)$ \\
\hline 1 & $36(44.44)$ \\
\hline$\geq 2$ & $13(16.05)$ \\
\hline Extranodal sites $>1$ & $7(8.60)$ \\
\hline \multicolumn{2}{|l|}{ Stage } \\
\hline IE & $42(51.90)$ \\
\hline IIE & $39(48.10)$ \\
\hline
\end{tabular}

LDH, lactate dehydrogenase; IPI, International Prognostic Index; NKPI, NK/T-cell lymphoma prognostic index.

\section{Response and relapse}

After chemotherapy, 9 patients had CR and 62 patients had PR, and the ORR was $87.7 \%$. After the completion of treatment, 68 patients had CR and the CR rate was $84 \%$. One patient had PR, and the ORR was $85.2 \%$. Three patients had PD during the treatment. The CR and ORR rates were $81 \%$ and $83.3 \%$ for stage IE, respectively, while the CR and ORR rates were both $87.2 \%$ for stage IIE.

The primary analysis was intention to treat and included 81 patients. After a median follow-up of 88.1 months, 3 patients $(3 / 81,3.7 \%)$ progressed during treatment, and 23 (23/81, 28.4\%) patients relapsed during the follow-up period, including 10 with local progression and 15 with systemic progression ( 5 with skin, 3 with central nervous system, 2 with lung, 2 with liver, and 1 with stomach). The estimated 5-year PFS was $63.4 \%$ (95\% CI, $52.5 \%$ to $76.4 \%$; Figure 1). Representative MRI images have been provided
Zhang et al. Efficacy and safety of DICEL in early ENKTL patients

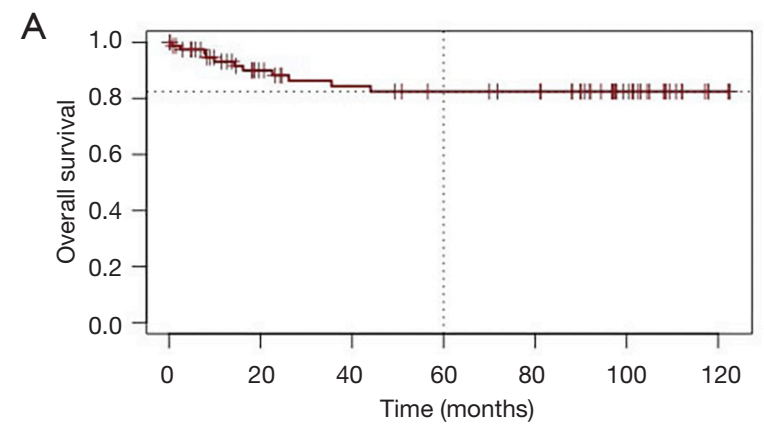

B

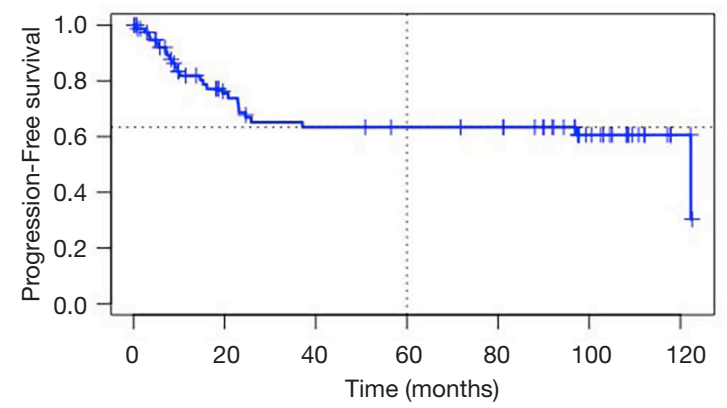

Figure 1 PFS and OS of stage I/IIE patients in our trial. KaplanMeier estimates of OS and PFS of patients treated with DICE-L chemotherapy. (A) The 5 -year OS of 81 patients was $82.4 \%$ (95\% CI, $73.4 \%$ to $92.6 \%$ ). The median follow-up time of survivors was 88.1 months. (B) The 5 -year PFS was $63.4 \%$ (95\% CI, $52.5 \%$ to $76.4 \%$ ). PFS, progression-free survival; OS, overall survival; DICE-L, L-asparaginase, dexamethasone, ifosfamide, cisplatin, and etoposide.

(Figure S1).

Nine patients (9/81) died of disease progression, and 1 patient (1/81) died of pneumonia during chemotherapy. The estimated 5-year OS was $82.4 \%$ (95\% CI, $73.4 \%$ to $92.6 \%$; Figure 1). There was no significant difference between stage IE and IIE, and the 5 -year OS rates were $83 \%$ in stage IE and $81.8 \%$ in stage IIE. A univariate analysis showed no significant association between OS and basic characteristics, such as age, stage, serum LDH, extranodal involvement sites, IPI, or NKPI. Moreover, the patients who achieved CR had a survival benefit, and the 5-year OS was $84.8 \%$. One patient who achieved PR received salvage chemotherapy and was still alive at last follow-up.

\section{Toxicity}

The adverse events are listed in Table 2. The most common toxicity was neutropenia (75.3\%), and most patients had 
Table 2 Patient toxicity profile after chemotherapy

\begin{tabular}{|c|c|c|}
\hline \multirow{2}{*}{ Toxicity } & \multicolumn{2}{|c|}{ Adverse event by grade } \\
\hline & 1 and 2 & 3 and 4 \\
\hline \multicolumn{3}{|l|}{ Hematological } \\
\hline Anemia & $12(14.8 \%)$ & $6(7.4 \%)$ \\
\hline Leucopenia & $6(7.4 \%)$ & $61(75.3 \%)$ \\
\hline Thrombocytopenia & $16(19.8 \%)$ & $19(23.5 \%)$ \\
\hline Febrile neutropenia & - & $17(21 \%)$ \\
\hline \multicolumn{3}{|l|}{ Non-hematological } \\
\hline ALT elevation & $8(9.9 \%)$ & $1(1.2 \%)$ \\
\hline Bilirubin elevation & $4(4.9 \%)$ & $1(1.2 \%)$ \\
\hline Amylase elevation & $6(7.4 \%)$ & - \\
\hline Creatinine elevation & - & $1(1.2 \%)$ \\
\hline Pancreatitis & - & $2(2.5 \%)$ \\
\hline Hyperglycemia & $1(1.2 \%)$ & $2(2.5 \%)$ \\
\hline Anorexia & - & $3(3.7 \%)$ \\
\hline Nausea & $11(13.6 \%)$ & $4(4.9 \%)$ \\
\hline Vomiting & $6(7.4 \%)$ & $4(4.9 \%)$ \\
\hline GI bleeding & - & $1(1.2 \%)$ \\
\hline Pneumonia & - & $3(3.7 \%)$ \\
\hline Hypoalbumin & $4(4.9 \%)$ & - \\
\hline Anaphylaxis & $2(2.5 \%)$ & $4(4.9 \%)$ \\
\hline Weight loss & $3(3.7 \%)$ & - \\
\hline Herpes zoster & - & $1(1.2 \%)$ \\
\hline Diarrhea & $3(3.7 \%)$ & - \\
\hline
\end{tabular}

ALT, alanine aminotransferase; GI, gastrointestinal.

grade 3 to 4 neutropenia (73.5\%). Additionally, 21\% (17 of 81 ) of patients suffered from febrile leucopenia. Three patients $(3.7 \%)$ experienced an $\mathrm{L}$-asparaginase related allergic reaction and 5 patients $(6.2 \%)$ experienced grade 1 to 2 amylase elevation leading to L-asparaginase being omitted from the next course of chemotherapy. No patients were diagnosed with pancreatitis due to amylase elevation. Nine patients (11.1\%) experienced grade 1 to 3 alanine aminotransferase (ALT) elevation. Four patients had grade 1 to 2 hypoalbumin. Twelve patients (14.8\%) had grade 2 to 3 nausea, which was controlled by supportive care without interrupting chemotherapy. One patient died of pneumonia after 1 cycle of DICE-L treatment.

\section{Discussion}

The optimal treatment strategy for early stage localized ENKTL has not been well defined. Our present study provides evidence that DICE-L followed by IMRT is an effective treatment for early stage ENKTL patients. The CR rate after the completion of treatment was $84 \%$, which was superior to the rates of $71.5-82.1 \%(15,16)$ reported in most previous concurrent chemoradiotherapy (CCRT) studies which combined the same chemotherapy regimens and L-asparaginase-based sequential chemoradiotherapy. It is also consistent with a CR rate of $86.5 \%$ with CCRT followed by L-asparaginase combined with VIPD (dexamethasone, ifosfamide, cisplatin and etoposide). It seems that L-asparaginase combined with DICE may be a good induction regimen for ENKTL treatment. This was also confirmed by a retrospective study which used the same regimen (17). In our study, 33 patients were treated with the DICE-L regimen followed by IMRT, and 26 patients (90.4\%) achieved CR (Table 3).

It is no doubt that radiotherapy is indispensable for localized ENKTL (24). Radiotherapy combined with chemotherapy improves the 5-year OS compared with chemotherapy or radiotherapy alone. In our present study, the CR rate after chemotherapy was only $8 \%$, but it reached to $84 \%$ after radiotherapy. Most patients with PR converted to CR because of radiotherapy. Radiotherapy and chemotherapy can be combined in different styles, for example, CCRT, sequential chemoradiotherapy with chemotherapy followed by radiotherapy, radiotherapy followed by chemotherapy, and sandwich chemoradiotherapy. The benefit of CCRT is improved local control and decreased distant progression, but the treatment related toxicity increases. Sequential chemoradiotherapy can overcome this disadvantage. According to a retrospective study, Oh et al. (19) showed that when effective chemotherapeutic regimens were adopted, such as SMILE(dexamethasone, methotrexate, ifosfamide, L-asparaginase, and etoposide), IMEP(ifosfamide, etoposide, methotrexate, and prednisolone), DeVIC (dexamethasone, etoposide, ifosfamide, and carboplatin), L-asparaginasebased, gemcitabine-based, ICE(etoposide, ifosfamide, and carboplatin or cisplatin), and ESHAP(etoposide, methylprednisolone, high-dose Ara-C, and cisplatin), no difference in CR rates and OS were noticed between CCRT and sequential chemoradiotherapy. In our study, sequential chemoradiotherapy with chemotherapy followed by radiotherapy showed a good outcome compared to most 
Table 3 Summary of CR, PFS, and OS from previous studies

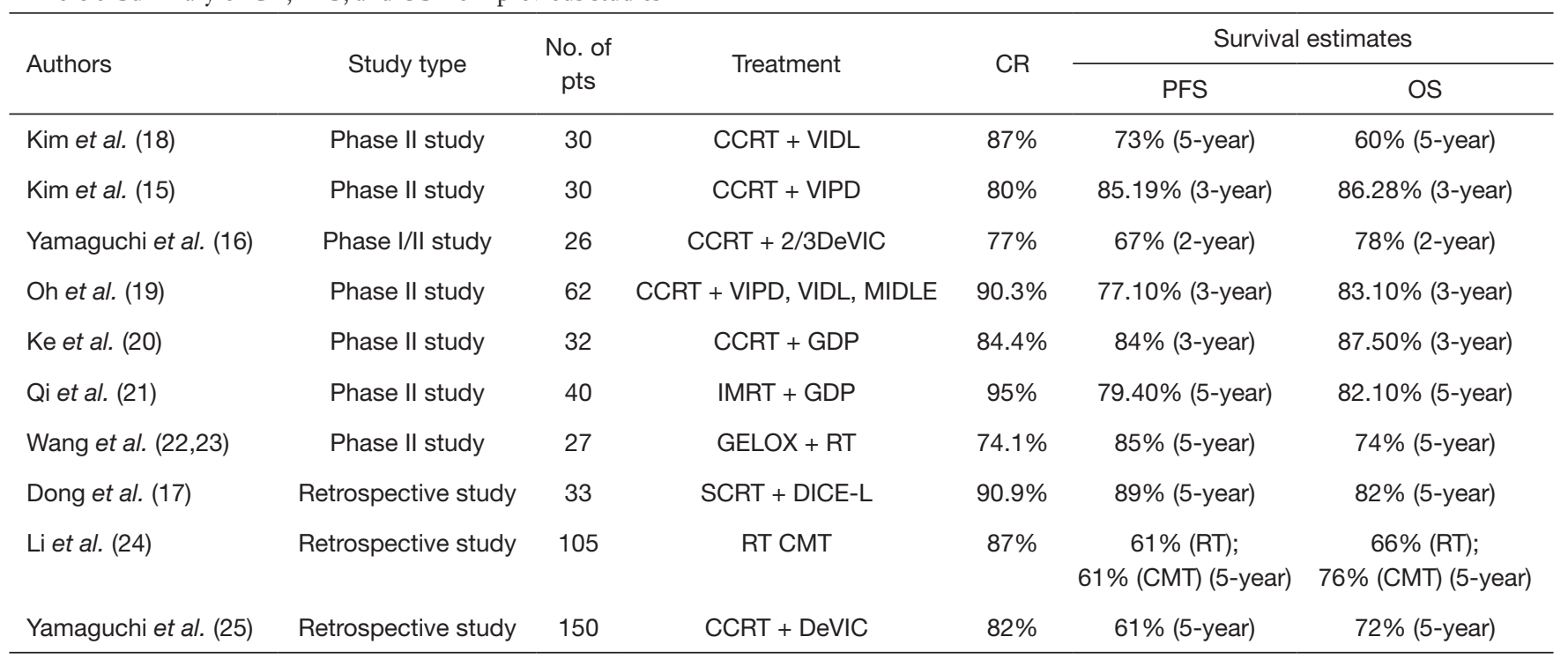

CR, complete response; PFS, progression-free survival; OS, overall survival; CCRT, concurrent chemoradiotherapy; VIDL, dexamethasone, ifosfamide, L-asparaginase, and etoposide; VIPD, dexamethasone, ifosfamide, and etoposide; DeVIC, dexamethasone, etoposide, ifosfamide, and carboplatin; MIDLE, methotrexate, etoposide, ifosfamide, mesna and L-asparaginase; GDP, gemcitabine, dexamethasone, and cisplatin; IMRT, intensity modulated radiation therapy; GELOX, gemcitabine, and oxaliplatin; RT, radiation therapy; SCRT, sequential chemoradiotherapy; DICE-L, L-asparaginase, dexamethasone, ifosfamide, cisplatin, and etoposide; CMT, combined-modality therapy. Major chemotherapies used in Li's study include CHOP (cyclophosphaminde, doxorubicin, vincristine, prednisone) or CHOP-bleo (CHOP + bleomycin). Nine had COBVP-16 (cisplatin, vincristine, bleomycin, prednisone), and 1 had COPP (cyclophosphaminde, vincristine, procarbazine, prednisone).

previous prospective trials (Table 3).

Chemotherapy is important for stage I/II patients, but the optimal chemotherapy regimen is unknown. Anthracyclinebased regimens, such as $\mathrm{CHOP}$, are disappointing due to the high $\mathrm{P}$-glycoprotein expression in tumor cells, and a 5 -year OS lower than 50\% (26). Non-anthracyclinebased chemotherapy regimens such as DICE can overcome multiple drug resistance and produced an exciting result when used as a CCRT regimen or adjuvant chemotherapy. Though good efficacy was achieved, increased toxicity also was acquired. Sequential chemoradiotherapy may lower the high toxicity caused by CCRT, but more chemotherapy cycles need to be given. L-asparaginase-based chemotherapy had a promising outcome as a second-line treatment with increased efficacy in localized ENKTL and reduced the number of chemotherapy cycles. Consequently, this decreased the toxicity. In our study, only 4 cycles of chemotherapy followed by IMRT produced a 5-year OS of $82.4 \%$. This is superior to most of the conducted prospective studies. It is also consistent with a previous study that used L-asparaginase, gemcitabine, and oxaliplatin (GELOX) treatment, which resulted in a 5-year OS of
$85 \%$. However, in this study, 6 cycles of chemotherapy were introduced.

Studies have shown that allergic reactions are common, with an incidence of $15-73 \%$ in L-asparaginase treatment, and this may decrease the efficacy. In our study, only $3.7 \%$ of patients experienced an allergic reaction (Table 4). This is lower than reported studies, perhaps due to the use of dexamethasone. In our chemotherapy combination, $40 \mathrm{mg}$ of dexamethasone was administered before every L-asparaginase transfusion. Polyethylene glycol-conjugated asparaginase (PEG-ASP), a long half time asparaginase which can decrease allergic reactions, was approved by the US Food and Drug Administration (FDA) for the treatment of acute lymphoblastic leukemia (ALL) patients allergic to L-asparaginase. It seems that PEG-ASP can be a substitute for L-asparaginase, but PEG-ASP has a high rate of hepatic toxicity, with ALT/AST elevation in 50-66.7\% of patients. Hypofibrinogenemia, hyperglycemia, and hypertriglyceridemia are common in PEG-ASP treatment compared to L-asparaginase treatment, and pancreatitis is also common in PEG-ASP treatment. In another prospective study using PEG-ASP combined with DICE, 
Table 4 Toxicity profile of different chemotherapy regimens

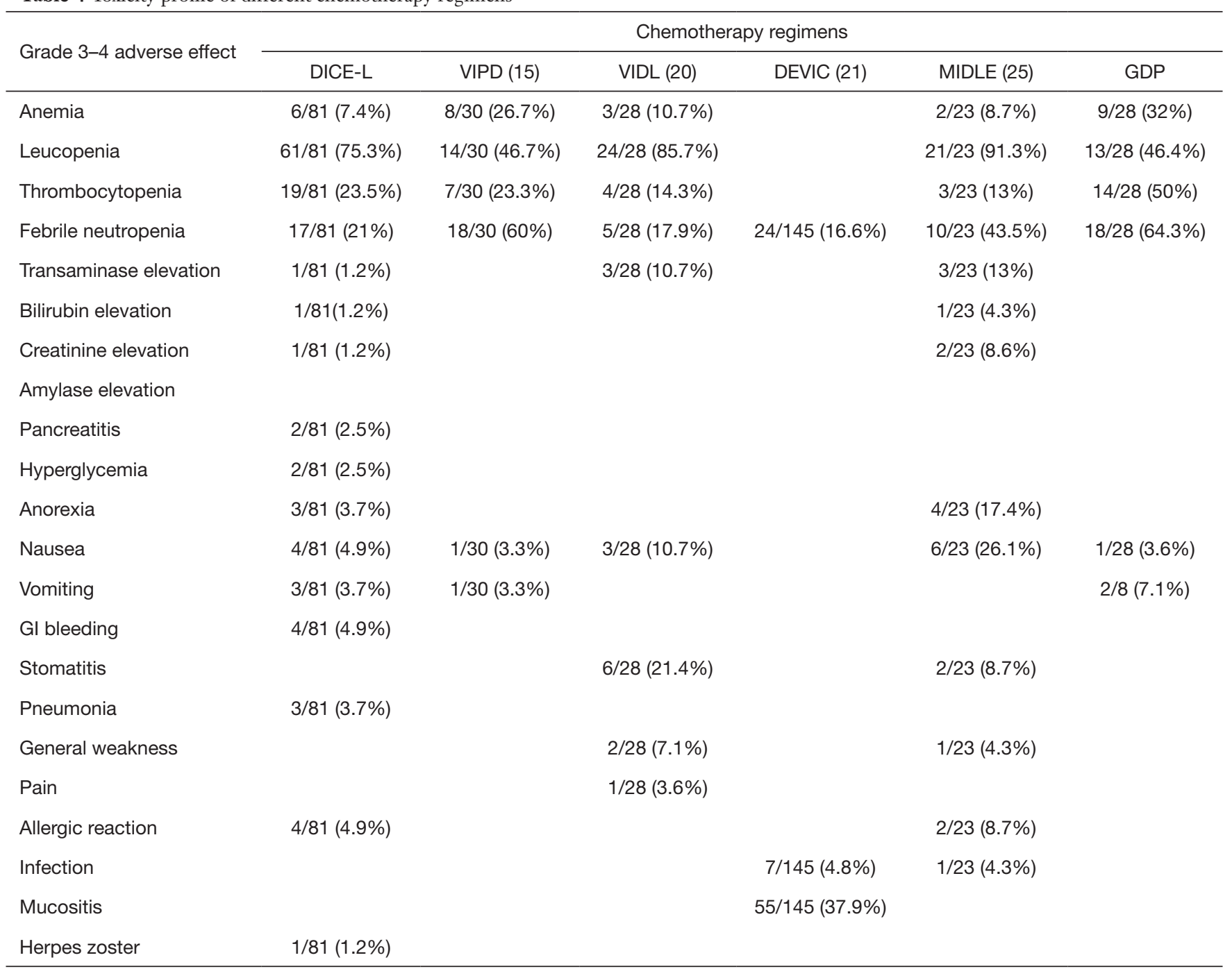

$36.5 \%$ of patients presented with increased ALT/AST, about $40 \%$ of patients had hyperbilirubinemia and decreased fibrinogen, and $90 \%$ of patients had hypoalbuminemia (10). In our present study, L-asparaginase combined with DICE had a low incidence of allergic reactions and less hepatic toxicity. As far as efficacy is concerned, there was no obvious difference between L-asparaginase and PEGASP. The CR and 2-year OS rates were, respectively, $81.6 \%$ and $80.1 \%$ for L-ASP combined with CHOP treatment versus $75.8 \%$ and $90.61 \%$ for PEG-ASP combined with CHOP treatment. The 5 -year OS was $82.4 \%$ in our present study with DICE-L and $87 \%$ in another study with DICEP (PEG-ASP, dexamethasone, ifosfamide, cisplatin and etoposide) treatment. Also, L-asparaginase combined with GEMOX had a 5 -year OS of $85 \%$ in a prospective study $(22,23)$. Thus, L-asparaginase is still a good choice for ENKTL, especially for patients who had severe hepatic toxicity under PEG-ASP treatment.

In the present study, though sequential chemoradiotherapy and a reduced number of chemotherapy cycles were introduced, hematological toxicity was still obvious, which was mostly due to the DICE combination. Most patients experienced dose reduction because of neutropenia or febrile neutropenia, but the outcome is still exciting. In Kim et al.'s study, cisplatin was omitted from the DICE regimen with a 5 -year OS of $73 \%$, which was compatible with the same design of DICE. Also, neutropenia can be compensated by G-CSF supplementation. 


\section{Page 8 of 9}

Hence, an optimal dosage and combination need to be determined to reduce hematological toxicity. In conclusion, DICE-L chemotherapy followed IMRT can be a feasible and effective treatment strategy for stage IE to IIE ENKTL with acceptable toxicity.

\section{Acknowledgments}

We wish to thank all the participants of this study. Funding: None.

\section{Footnote}

Reporting Checklist: The authors have completed the TREND reporting checklist. Available at https://dx.doi. org/10.21037/atm-21-3525

Data Sharing Statement: Available at https://dx.doi. org/10.21037/atm-21-3525

Conflicts of Interest: All authors have completed the ICMJE uniform disclosure form (available at https://dx.doi. org/10.21037/atm-21-3525). The authors have no conflicts of interest to declare.

Ethical Statement: The authors are accountable for all aspects of the work in ensuring that questions related to the accuracy or integrity of any part of the work are appropriately investigated and resolved. The study was approved by the institute review board of Shanghai Cancer Center of Fudan University (No. 090674-1) and was first registered on $6^{\text {th }}$ July 2009. The NCT number is NCT00933673. Written informed consent was obtained from all patients. If patients were under 18 , consent was obtained from a parent and/or legal guardian. The study was conducted in accordance with the principles of the Declaration of Helsinki (as revised in 2013).

Open Access Statement: This is an Open Access article distributed in accordance with the Creative Commons Attribution-NonCommercial-NoDerivs 4.0 International License (CC BY-NC-ND 4.0), which permits the noncommercial replication and distribution of the article with the strict proviso that no changes or edits are made and the original work is properly cited (including links to both the formal publication through the relevant DOI and the license). See: https://creativecommons.org/licenses/by-nc-nd/4.0/.

\section{Zhang et al. Efficacy and safety of DICEL in early ENKTL patients}

\section{References}

1. Jaffe ES, Chan JK, Su IJ, et al. Report of the Workshop on Nasal and Related Extranodal Angiocentric T/Natural Killer Cell Lymphomas. Definitions, differential diagnosis, and epidemiology. Am J Surg Pathol 1996;20:103-11.

2. Li X, Li G, Gao Z, Zhou X, Zhu X. The relative frequencies of lymphoma subtypes in China: A nationwide study of 10002 cases by the Chinese Lymphoma Study Group. Ann Oncol. 2011;22:iv141.

3. Fox CP, Shannon-Lowe C, Rowe M. Deciphering the role of Epstein-Barr virus in the pathogenesis of $\mathrm{T}$ and $\mathrm{NK}$ cell lymphoproliferations. Herpesviridae 2011;2:8.

4. Chim CS, Ma SY, Au WY, et al. Primary nasal natural killer cell lymphoma: long-term treatment outcome and relationship with the International Prognostic Index. Blood 2004;103:216-21.

5. Li CC, Tien HF, Tang JL, et al. Treatment outcome and pattern of failure in 77 patients with sinonasal natural killer/ T-cell or T-cell lymphoma. Cancer 2004;100:366-75.

6. Proulx GM, Caudra-Garcia I, Ferry J, et al. Lymphoma of the nasal cavity and paranasal sinuses: treatment and outcome of early-stage disease. Am J Clin Oncol 2003;26:6-11.

7. Bao C, Zhou D, Zhu L, et al. Increased serum level of interleukin-6 correlates with negative prognostic factors in extranodal NK/T-cell lymphoma. Transl Cancer Res 2020;9:2378-89.

8. Avilés A, Neri N, Fernández R, et al. Combined therapy in untreated patients improves outcome in nasal NK/ T lymphoma: results of a clinical trial. Med Oncol 2013;30:637.

9. Lee J, Suh C, Park YH, et al. Extranodal natural killer T-cell lymphoma, nasal-type: a prognostic model from a retrospective multicenter study. J Clin Oncol 2006;24:612-8.

10. Liu Y, Xue K, Xia Z, et al. Radiotherapy followed by DICEP regimen in treatment of newly diagnosed, stage IE/IIE, extranodal NK/T-cell lymphoma patients. Cancer Med 2020;9:5400-5.

11. Yamaguchi M, Tobinai K, Oguchi M, et al. Concurrent chemoradiotherapy for localized nasal natural killer/ T-cell lymphoma: an updated analysis of the Japan clinical oncology group study JCOG0211. J Clin Oncol 2012;30:4044-6.

12. Yamaguchi M, Kwong YL, Kim WS, et al. Phase II study of SMILE chemotherapy for newly diagnosed stage IV, relapsed, or refractory extranodal natural killer (NK)/T-cell 
lymphoma, nasal type: the NK-Cell Tumor Study Group study. J Clin Oncol 2011;29:4410-6.

13. Cheson BD, Pfistner B, Juweid ME, et al. Revised response criteria for malignant lymphoma. J Clin Oncol 2007;25:579-86.

14. Kim BS, Kim TY, Kim CW, et al. Therapeutic outcome of extranodal NK/T-cell lymphoma initially treated with chemotherapy--result of chemotherapy in NK/T-cell lymphoma. Acta Oncol 2003;42:779-83.

15. Kim SJ, Kim K, Kim BS, et al. Phase II trial of concurrent radiation and weekly cisplatin followed by VIPD chemotherapy in newly diagnosed, stage IE to IIE, nasal, extranodal NK/T-Cell Lymphoma: Consortium for Improving Survival of Lymphoma study. J Clin Oncol 2009;27:6027-32.

16. Yamaguchi M, Tobinai K, Oguchi M, et al. Phase I/II study of concurrent chemoradiotherapy for localized nasal natural killer/T-cell lymphoma: Japan Clinical Oncology Group Study JCOG0211. J Clin Oncol 2009;27:5594-600.

17. Dong LH, Zhang LJ, Wang WJ, et al. Sequential DICE combined with 1-asparaginase chemotherapy followed by involved field radiation in newly diagnosed, stage IE to IIE, nasal and extranodal NK/T-cell lymphoma. Leuk Lymphoma 2016;57:1600-6.

18. Kim SJ, Yang DH, Kim JS, et al. Concurrent chemoradiotherapy followed by L-asparaginase-containing chemotherapy, VIDL, for localized nasal extranodal NK/T cell lymphoma: CISL08-01 phase II study. Ann Hematol 2014;93:1895-901.

19. Oh D, Ahn YC, Kim SJ, et al. Concurrent Chemoradiation Therapy Followed by Consolidation Chemotherapy for Localized Extranodal Natural Killer/T-Cell Lymphoma, Nasal Type. Int J Radiat Oncol Biol Phys 2015;93:677-83.

Cite this article as: Zhang Y, Liu Y, Xia Z, Jin J, Xue K, Wang J, Sun H, Lv F, Liu X, Cao J, Hong X, Guo Y, Ma X, Zhang Q. Phase II study of sequential chemoradiotherapy with L-asparaginase, dexamethasone, ifosfamide, cisplatin, and etoposide (DICE-L) in the early stage of extranodal natural killer (NK)/T-cell lymphoma. Ann Transl Med 2021;9(14):1178. doi: $10.21037 / \mathrm{atm}-21-3525$
20. Ke QH, Zhou SQ, Du W, et al. Concurrent IMRT and weekly cisplatin followed by GDP chemotherapy in newly diagnosed, stage IE to IIE, nasal, extranodal NK/T-Cell lymphoma. Blood Cancer J 2014;4:e267.

21. Qi F, Wang WH, He XH, et al. Phase 2 Study of Firstline Intensity Modulated Radiation Therapy Followed by Gemcitabine, Dexamethasone, and Cisplatin for HighRisk, Early Stage Extranodal Nasal-Type NK/T-Cell Lymphoma: The GREEN Study. Int J Radiat Oncol Biol Phys 2018;102:61-70.

22. Wang L, Wang ZH, Chen XQ, et al. Firstline combination of gemcitabine, oxaliplatin, and L-asparaginase (GELOX) followed by involved-field radiation therapy for patients with stage IE/IIE extranodal natural killer/T-cell lymphoma. Cancer 2013;119:348-55.

23. Wang L, Wang ZH, Chen XQ, et al. First-line combination of GELOX followed by radiation therapy for patients with stage IE/IIE ENKTL: An updated analysis with long-term follow-up. Oncol Lett 2015;10:1036-40.

24. Zhang L, Wei Y, Yan X, et al. Survivin is a prognostic marker and therapeutic target for extranodal, nasaltype natural killer/T cell lymphoma. Ann Transl Med 2019;7:316.

25. Yamaguchi M, Suzuki R, Oguchi M, et al. Treatments and Outcomes of Patients With Extranodal Natural Killer/ T-Cell Lymphoma Diagnosed Between 2000 and 2013: A Cooperative Study in Japan. J Clin Oncol 2017;35:32-9.

26. Ribrag V, Ell Hajj M, Janot F, et al. Early locoregional high-dose radiotherapy is associated with long-term disease control in localized primary angiocentric lymphoma of the nose and nasopharynx. Leukemia 2001;15:1123-6.

(English Language Editor: C Betlzar) 


\section{Supplementary}

A. Pretreatment

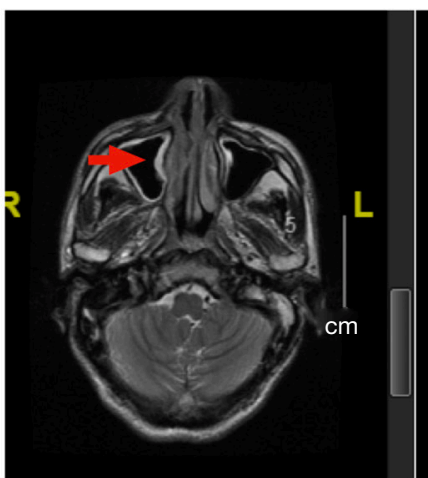

B. After two cycles of DICE-L

C. After four cycles of DICE-L

D. After IMRT

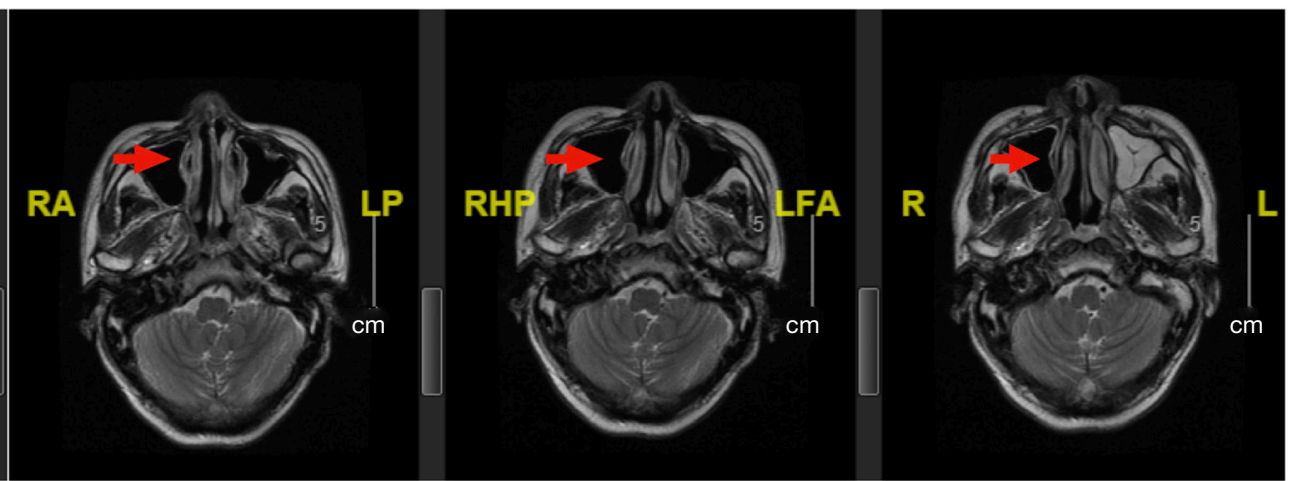

Figure S1 A 44-year-old man diagnosed with Stage IE ENKTL. MRI of the Nasal area. Red arrow represented the lesion in the nasal area. 